\title{
BMJ Open Prevalence and incidence of pulmonary hypertension among HIV-infected people in Africa: a systematic review and meta-analysis
}

Jean Joel R Bigna, ${ }^{1,2}$ Jobert Richie N Nansseu, ${ }^{3,4}$ Lewis N Um, ${ }^{5}$ Steve Raoul N Noumegni, ${ }^{5}$ Paule Sandra D Simé, ${ }^{5}$ Leopold Ndemngue Aminde, ${ }^{6,7}$ Sinata Koulla-Shiro, ${ }^{5,8}$ Jean Jacques N Noubiap ${ }^{9,10}$

To cite: Bigna JJR, Nansseu JRN, Um LN, et al. Prevalence and incidence of pulmonary hypertension among HIV-infected people in Africa: a systematic review and meta-analysis. BMJ Open 2016;6:e011921.

doi:10.1136/bmjopen-2016011921

- Prepublication history and additional material is available. To view please visit the journal (http://dx.doi.org/ 10.1136/bmjopen-2016011921).

Received 15 March 2016 Revised 30 July 2016 Accepted 3 August 2016

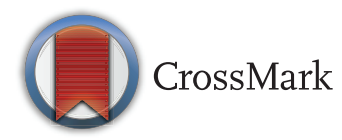

For numbered affiliations see end of article.

Correspondence to Dr Jean Joel R Bigna; bignarimjj@yahoo.fr

\section{ABSTRACT}

Objective: Patients infected with HIV have a direly increased risk of developing pulmonary hypertension $(\mathrm{PH})$, and of dying from the condition. While Africa carries the greatest burden of HIV infection worldwide, there is unclear data summarising the epidemiology of $\mathrm{PH}$ among HIV-infected people in this region. Our objective was to determine the prevalence and incidence of $\mathrm{PH}$ among HIV-infected people living across Africa.

Design: A systematic review and meta-analysis. Participants: HIV-infected African people residing in Africa.

Outcome: Prevalence and incidence of $\mathrm{PH}$ diagnosed through echocardiography or right heart catheterisation.

Data sources: Articles published in PubMed/ MEDLINE, EMBASE, African Journals Online and African Index Medicus between 1 January 1980 and 30 June 2016, without any language restriction. Results: Overall, 121 studies were screened; 3 were included in this review: 1 from Southern Africa (South Africa), 1 from Eastern Africa (Tanzania) and 1 from Central Africa (Cameroon). These studies included HIV-infected adult patients selected based on presentation with cardiovascular symptoms. No study reported $\mathrm{PH}$ incidence or $\mathrm{PH}$ incidence/prevalence among children and adolescents. The quality assessment yielded moderate risk of bias. Ages of participants ranged between 18 and 78 years, and the proportion of females varied between $52.3 \%$ and $68.8 \%$. The prevalence of $\mathrm{PH}$ in the pooled sample of 664 patients was $14 \%(95 \% \mathrm{Cl} 6 \%-23 \%)$.

Limitations: Only 3 studies were found eligible from 3 regions of the African continent.

Conclusions: The prevalence of PH among HIVinfected people in Africa seems very high. Further studies are urgently warranted to determine the incidence of HIV-induced PH, which must include all subregions of Africa.

Trial registration number: Review registration number PROSPERO CRD42016033863.

\section{Strengths and limitations of this study}

- Only three studies were eligible from three of the five subregions of Africa.

- It was impossible to disaggregate data between patients on antiretroviral treatment and those naïve of antiretroviral treatment.

- This is the first systematic review and meta-analysis summarising the prevalence of pulmonary hypertension among HIV-infected people in Africa.

- Strong and robust statistical methods were used, which permitted to figure out an elevated relative prevalence of pulmonary hypertension in HIV-infected individuals residing in Africa.

\section{INTRODUCTION}

HIV continues to be a major global public health issue, having hampered more than 34 million lives so far. There were $\sim 36.9$ million people living with HIV at the end of 2014, with 2 million people becoming newly infected worldwide; what is worse, 1.2 million people died from HIV-related consequences in 2014. Sub-Saharan Africa (SSA) is the most affected region with 25.8 million people living with HIV in 2014. Besides, SSA accounts for almost $70 \%$ of the global HIV new infections. ${ }^{12}$

Pulmonary hypertension $(\mathrm{PH})$ is defined as a mean pulmonary arterial pressure $(\mathrm{mPAP}) \geq 25 \mathrm{~mm} \mathrm{Hg}$ on right heart catheterisation at rest. ${ }^{3} 4$ The classification of $\mathrm{PH}$ includes pulmonary arterial hypertension $(\mathrm{PAH}), \mathrm{PH}$ due to left heart diseases, $\mathrm{PH}$ due to respiratory diseases and/or hypoxaemia, $\mathrm{PH}$ due to chronic thromboembolic disease, and $\mathrm{PH}$ due to unclear multifactorial mechanisms. ${ }^{3}$ To date, evidence has accumulated a causal relationship between HIV 
infection and $\mathrm{PAH}^{5}$ The pathogenesis of $\mathrm{PAH}$ is complex. It is currently admitted that this may probably result from the interaction between multiple modulating genes and environmental factors. The physiopathology includes (1) increase in cytokine secretion inducing a dysregulation of endothelial and vascular smooth muscle cell growth and an imbalance between endogenous vasodilators and constrictors; (2) proliferation of HIV viral proteins leading to vascular oxidative stress, smooth myocyte proliferation and migration, and endothelial injury; and (3) genetic predisposition due to some major histocompatibility complex alleles, particularly HDL-DR6 and HLA-DR5. ${ }^{5}$

HIV-infected patients have a greater incidence of $\mathrm{PH}$ and almost a 2500-fold increased risk of developing the condition in comparison with the general population (incidence of idiopathic PAH: 1-2 per million persons). ${ }^{6}{ }^{7}$ Furthermore, the development of PAH in HIV-infected individuals reduces their probability of survival by half when compared with their counterparts without HIV-induced PAH. About two-thirds of deaths in patients with HIV-induced PAH are due to the consequences of $\mathrm{PAH}$ such as: right heart failure, cardiogenic shock and sudden death. ${ }^{8}$

While Africa carries the greatest burden of HIV infection globally, ${ }^{1}{ }^{2}$ there are unclear data summarising the epidemiology of PH in HIV-infected populations. To fill this critical gap, we conducted a systematic review and meta-analysis, with the objective to determine the prevalence and incidence of $\mathrm{PH}$ among African HIV-infected people residing in Africa.

\section{METHODS}

The Preferred Reporting Items for Systematic reviews and Meta-Analyses guidelines served as the template for reporting the present review. ${ }^{9}$ The Preferred Reporting Items for Systematic reviews and Meta-Analyses checklist can be found in online supplementary appendix S1. This review was registered in the PROSPERO International Prospective Register of systematic reviews, registration number CRD42016033863.

\section{Eligibility criteria for considering studies to include in the review Inclusion criteria}

- Cross-sectional, case-control or cohort studies of HIV-infected people residing in African countries which have reported the prevalence or incidence of $\mathrm{PH}$, or enough data to compute these estimates.

- Studies in which diagnosis of PH was based on right heart catheterisation finding a mPAP $\geq 25 \mathrm{~mm} \mathrm{Hg}$ or abnormal echocardiography examination for a pulmonary arterial systolic pressure (PASP) $>35 \mathrm{~mm} \mathrm{Hg} .{ }^{34}$

- We considered all published and unpublished studies reported from 1 January 1980 to 30 June 2016. No language restriction was applied.
Exclusion criteria

- Studies conducted among populations of African origin residing outside Africa.

- Studies not performed in human participants.

- Studies in subgroups of participants selected on the basis of presence of $\mathrm{PH}$.

- Case series, reviews, letters, commentaries and editorials.

- Studies lacking primary data and/or explicit description of methods.

For duplicate reports, the most comprehensive and up-to-date version was considered for this review.

\section{Search strategy for identifying relevant studies}

The search strategy was implemented in two stages:

\section{Bibliographic database search}

We performed a comprehensive and exhaustive search of PubMed/MEDLINE, Excerpta Medica Database (EMBASE), African Journals Online and African Index Medicus to identify all relevant articles published on $\mathrm{PH}$ among HIV-infected people in Africa between 1 January 1980 and 30 June 2016, without any language restriction. We conceived and applied a search strategy based on the combination of relevant terms and names of each of the 54 African countries and African subregions. We used the following terms for PH: 'pulmonary hypertension' and 'pulmonary arterial hypertension'. For HIV, we used the terms 'HIV' and 'AIDS'. The last electronic search was carried out on 1 July 2016. The main search strategy conducted in PubMed is shown in online supplementary appendix S2. This search strategy was adapted to fit with other databases.

\section{Searching other sources}

We conducted manual searches which consisted in scanning the reference lists of eligible papers and other relevant review articles, specialist journals and conference proceedings (Advances in Pulmonary Hypertension; Pulmonary Hypertension Association's International PH Conference, Pulmonary Hypertension Association-Ireland's Annual Conference, Annual Scientific Meeting of the Pulmonary Hypertension Society of Australia and New Zealand, and Annual Joint Pulmonary Hypertension).

\section{Selection of studies for inclusion in the review}

Two investigators (JJRB and LNU) independently identified articles and sequentially screened their titles and abstracts for eligibility. Full texts of articles deemed potentially eligible were acquired. These investigators further independently assessed the full text of each study for eligibility, and consensually retained studies to be included. Disagreements when existing were solved by a third author (JRNN). We used a screening guide to ensure that the selection criteria were reliably applied by all review authors. Agreement was measured using the statistic. $^{10}$ 


\section{Data extraction and management}

Two investigators (JJRB and LNU) independently extracted data pertaining to general information (authors, year, country, region), study characteristics (study design, setting, sample size, mean or median age and proportion of female participants, diagnosis criteria for $\mathrm{PH}$, antiretroviral therapy (ART)), and prevalence and/or incidence of $\mathrm{PH}$. Where only primary data (sample size and number of outcomes) were provided, these data were used to calculate the prevalence or incidence estimates. Data were extracted using a preconceived and standardised data abstraction form. Disagreements between authors were reconciled through discussion and consensus, or arbitration by a third author (JRNN) whenever necessary. Agreement was measured using the $\mathrm{\kappa}$ statistic. ${ }^{10}$

\section{Appraisal of the quality of included studies}

Two investigators (JJRB and LNU) evaluated included studies for methodological quality and risk of bias using an adapted version of the Risk of Bias Tool for Prevalence Studies developed by Hoy et $a l^{11}$ (see online supplementary appendix S3). Furthermore, the reporting quality of each study was assessed using the STROBE checklist (see online supplementary appendix S4). ${ }^{12}$ Agreement was measured using the $\kappa$ statistic. ${ }^{10}$

\section{Data synthesis and analysis}

Data were analysed using STATA V.13.0 for Windows. Forest plots were drawn to visualise the combined prevalence and extent of heterogeneity between studies. Owing to the clinical differences across patients included in the studies (difference in the clinical presentation of patients with cardiac symptoms, inconsistency regarding ART), a random-effects meta-analysis was used to pool prevalence data, ${ }^{13}$ after stabilising the variance of individual studies using the Freeman-Tukey double arc-sine transformation. ${ }^{14}$ Heterogeneity was assessed using the $\chi^{2}$ test on Cochrane's $Q$ statistic, ${ }^{15}$ and quantified by calculating the $\mathrm{I}^{2}$ (with values of $25 \%, 50 \%$ and $75 \%$ being representative of low, medium and high heterogeneity, respectively). ${ }^{16}$ In order to assess possible publication bias, Egger weighted regression methods were used. ${ }^{17} \mathrm{~A} \mathrm{p}$ value $<0.05$ was considered indicative of statistically significant publication bias.

\section{RESULTS}

\section{Characteristics of included studies}

Initially, a total of 121 articles were identified (figure 1). After elimination of duplicates, screening titles and abstracts, 106 papers were found completely irrelevant and excluded. Agreement between investigators on abstract selection was high $(\kappa=0.90, \mathrm{p}<0.001$; given a maximum attainable $\kappa=1$ ). Full texts of the remaining 15 studies were scrutinised for eligibility, among which 12 studies were excluded. There was no disagreement between investigators for full-text selection. In two of these excluded studies, the cut-off for diagnosis of $\mathrm{PH}$ was $25 \mathrm{~mm} \mathrm{Hg}$, rather than $35 \mathrm{~mm} \mathrm{Hg}$ on echocardiography. ${ }^{18}{ }^{19}$ Concerning these two studies, the prevalence of PH was $6.9 \%$ among adolescents in Zimbabwe, ${ }^{18}$ and $4 \%$ among adults in Nigeria. ${ }^{19}$ Overall, three studies were found eligible; hence they were included in the meta-analysis (figure 1).

All the three studies reported the prevalence of $\mathrm{PH}$ without any analysis with regard to the patients' ART

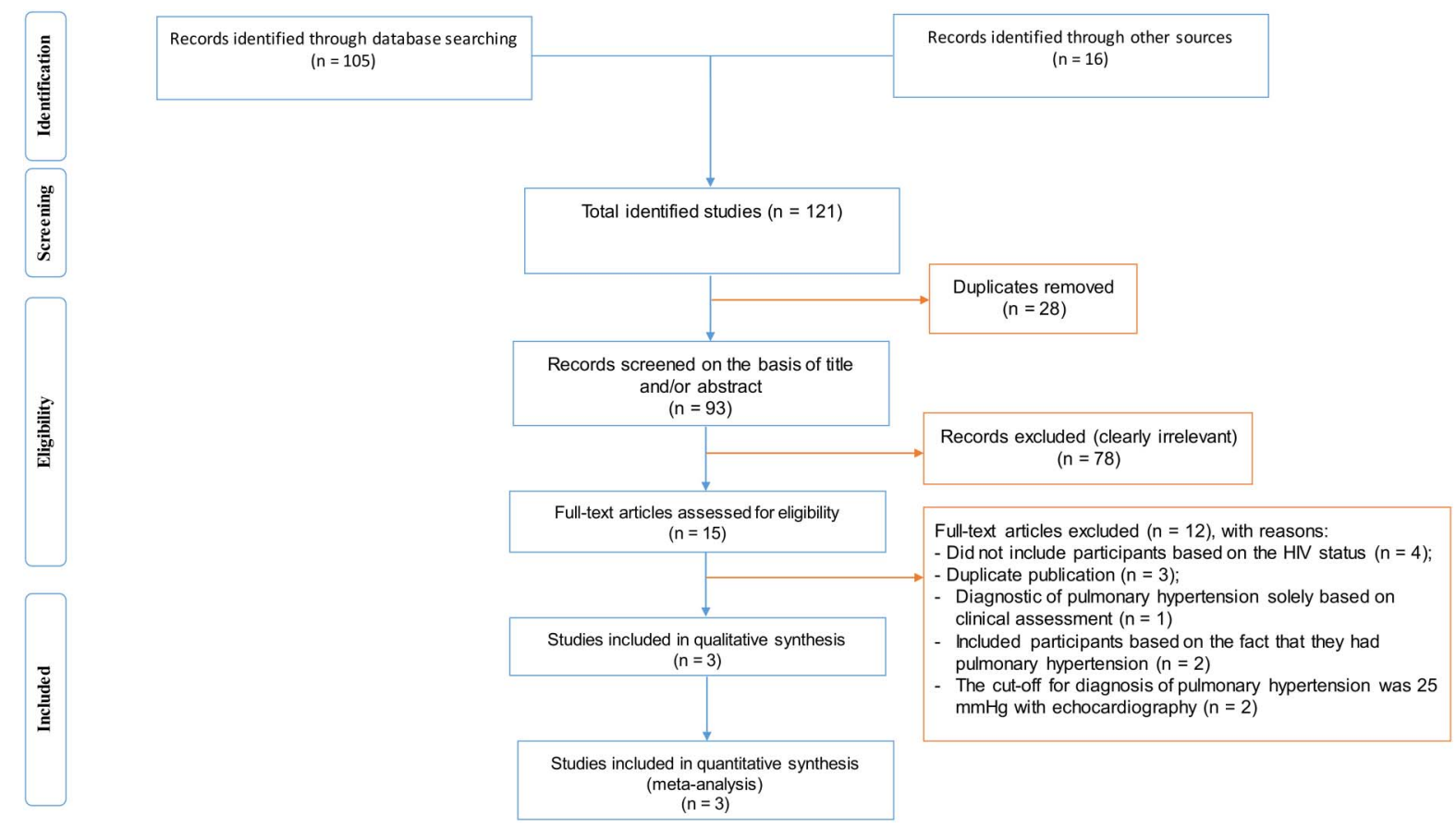

Figure 1 Process of identification and selection of studies for inclusion in the review (PRISMA flow diagram). PRISMA, Preferred Reporting Items for Systematic reviews and Meta-Analyses. 
regimens and none reported the incidence of $\mathrm{PH}$. The studies were reported from Cameroon, Tanzania and South Africa; there was no study from the Western and Northern parts of Africa. They were conducted between 2006 and 2014, published between 2012 and 2015 and included HIV-adult patients who were selected based on presentation with cardiovascular symptoms. Characteristics of these studies are summarised in table 1 . The female proportion varied from $52.3 \%$ to $68.8 \%$ (table 1). For one of these studies, ${ }^{20}$ we calculated the overall prevalence since it separately reported the prevalence for men and that for women. For the two other studies, we reported the prevalence of $\mathrm{PH}$ as it appeared in the studies. ${ }^{21} 22$

Considering STROBE guidelines, the quality of reporting was good/fair for all included studies. These studies had a moderate risk of bias (table 2). Agreement between investigators on quality assessment of studies was high $(\kappa=0.88, \mathrm{p}<0.001$; given a maximum attainable $\kappa=1)$.

\section{Pooled prevalence of PH among HIV-infected people in Africa}

There was a wide variation in $\mathrm{PH}$ prevalence (figure 2). The heterogeneity was high $\left(\mathrm{I}^{2}=81.4 \%, \mathrm{p}<0.001\right)$. The prevalence of $\mathrm{PH}$ in the pooled sample of 664 individuals was $14 \%$ (95\% CI $6 \%$ to $23 \%$ ). No evidence of publication bias was observed (figure 3); results of Egger's weighted regression test did not show any bias of publication $(\mathrm{t}=4.09, \mathrm{p}=0.153)$.

\section{DISCUSSION}

These moderate risk of bias studies conducted between 2006 and 2014 from the three WHO regions of Africa showed a pooled $14 \%(95 \%$ CI $6 \%-23 \%)$ prevalence of $\mathrm{PH}$ among African HIV-infected adults. The prevalence of PH among HIV-infected people in Africa found in this meta-analysis is close to what was reported in a recent narrative review (5-13\%) targeting the same population. ${ }^{5}$ This prevalence in SSA is notably higher when compared with that from developed countries, around $0.5 \% .^{723-25}$ Certain reasons can explain this large discrepancy observed. Access to and retention in care is lower in low-income and middle-income countries (including most of African countries), leading to late diagnosis and management of the HIV infection. Consequently, patients will probably arrive at a more advanced stage of disease progression. Besides, ART initiation in developed countries does not take into consideration the CD4-cell count and HIV clinical stage in contrast to the developing world where these parameters are still taken into account, leading to initiation of ART perhaps when processes towards HIV-induced complications such as $\mathrm{PH}$ have already started. ${ }^{26-29}$

However, it is hoped that the new WHO recommendations for initiating ART regardless of CD4 count ('test and treat' policy) will probably lead to a change in the epidemiology of PH in HIV-infected people in Africa. ${ }^{30}$

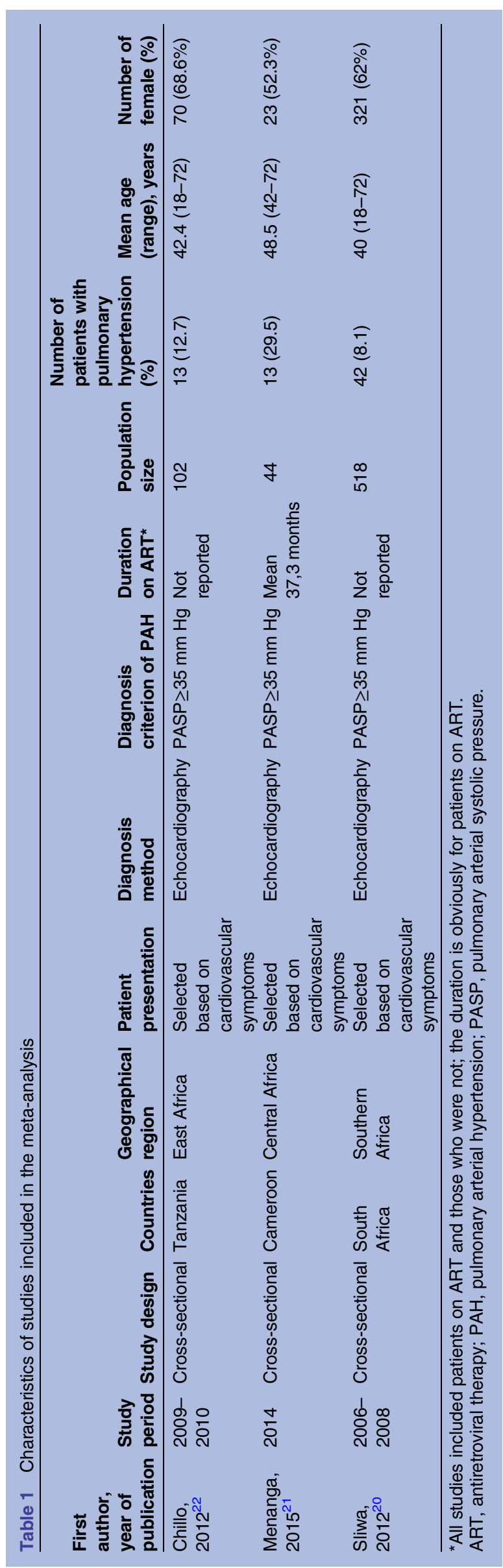


Table 2 Quality assessment of included studies

\begin{tabular}{|c|c|c|c|c|}
\hline & \multicolumn{2}{|c|}{ STROBE quality of reporting* } & \multicolumn{2}{|c|}{ Risk of bias by Hoy et $a I^{11} t$} \\
\hline & Score range, 0-22 & Interpretation & Score range, 0-10 & Interpretation \\
\hline Chillo et $a^{22}$ & 17 & Good/fair quality & 7 & Moderate risk \\
\hline Menanga et $a^{21}$ & 19 & Good/fair quality & 7 & Moderate risk \\
\hline Sliwa et $a^{R^{0}}$ & 19 & Good/fair quality & 6 & Moderate risk \\
\hline
\end{tabular}

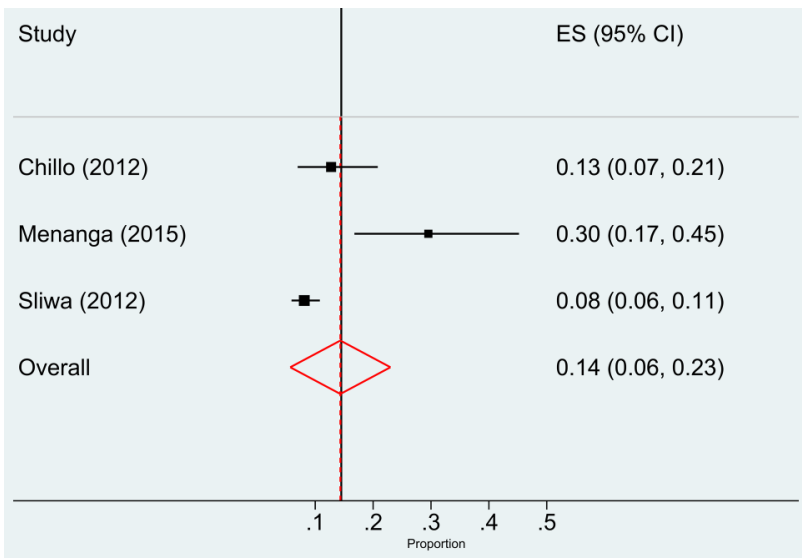

Figure 2 Forest plots of pulmonary hypertension prevalence among HIV-infected people in Africa. ES, estimated size.

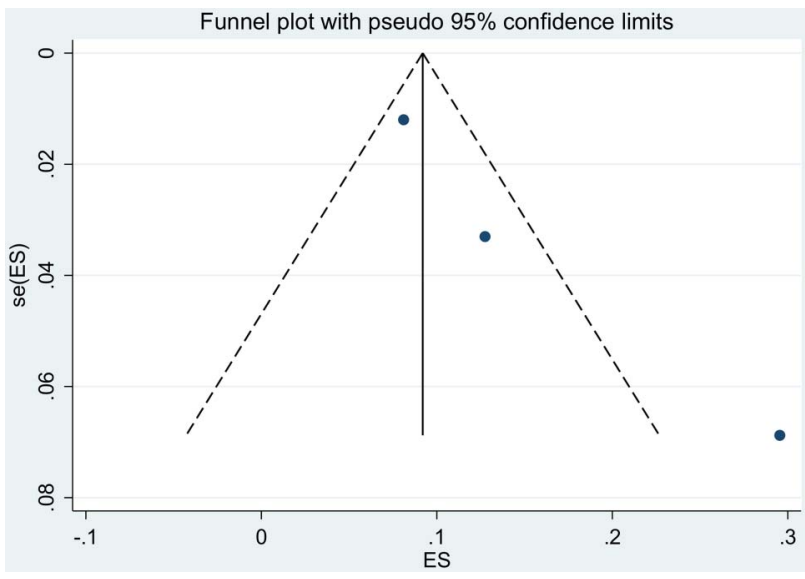

Figure 3 Funnel plots of SEs of pulmonary hypertension prevalence among HIV-infected people in Africa. ES, estimated size; se (ES), SE of estimated size.

Indeed, it may lead to a decrease in the prevalence of $\mathrm{PH}$ in this group of patients. ${ }^{31}$

For now, there is no cure for PAH. ${ }^{32}$ Nonetheless, the use of highly active antiretroviral therapy (HAART), whose aim is to prevent the multiplication of the virus and thus make undetectable the viral load could considerably reduce the risk of development or progression of $\mathrm{PH}$ in HIV-infected patients. It has been demonstrated that the detection of plasma HIV-RNA was associated with an increased risk of developing $\mathrm{PH}^{23}$ This also suggests that early initiation of HAART may prevent or delay
$\mathrm{PH}$ apparition in HIV-infected individuals, though data in this respect are controversial. ${ }^{33}$ In any case, HAART should not be used as single therapy for HIV-induced $\mathrm{PAH}$; early initiation of PAH-specific management is of paramount importance. In fact, HAART only ameliorates the outcome of patients with HIV-induced PAH. ${ }^{5}$

Two interesting studies were excluded from our meta-analysis because the authors diagnosed $\mathrm{PH}$ with a cut-off of $25 \mathrm{~mm} \mathrm{Hg}$ for pulmonary arterial systolic pressure using echocardiography. ${ }^{18} 19$ We suggest researchers and clinicians to adhere to the updated guidelines for the proper diagnosis of $\mathrm{PH}$.

\section{Limitations}

Our study presents some flaws. First, our study was based on a limited number of published studies. Nonetheless, we explored four databases. Second, concerning the quality of evidence, the risk of bias of included studies was moderate suggesting that further research is likely to have an important impact on our confidence in the estimate, and may change this estimate. Although there is no publication bias, this study may lack power due to the limited number of included studies. Third, considering that almost all studies included were hospital-based, it is likely that the pooled prevalence of $\mathrm{PH}$ we obtained is symptom-driven. Moreover, none of the studies included in this review assessed the incidence of HIV-induced $\mathrm{PAH}$ as well as the relationship between the condition and ART (difference between ART+ and ART- patients, the specific regimens increasing the risk, and duration of ART at which PH is likely to occur). Meta-analysis of small number of studies can be questionable; notwithstanding, it gives an overview of the burden of $\mathrm{PH}$ among HIV-infected people in Africa. Therefore, highquality observational studies are warranted to fill these crucial gaps. The ongoing PAPUCO cohort study is welcome for bringing some scientific information regarding $\mathrm{PH}$ in African countries. ${ }^{34}$

\section{CONCLUSIONS}

The prevalence of $\mathrm{PH}$ among people living with HIV in Africa seems very high, which may significantly contribute to the elevated HIV-related mortality rate witnessed all round the continent. Therefore, healthcare providers must be aware of this reality, and potentially undertake regular screening of $\mathrm{PH}$ among their $\mathrm{HIV}$-infected patients, 
and management tailored accordingly for those diagnosed with the condition. Besides, it should be discussed early during initiation of HAART in African countries to curtail the burden of HIV-induced PAH as well as other HIV-related complications. The new 2015 WHO guidelines on initiation of ART regardless of $\mathrm{CD} 4$ count, age and HIV disease progression are likely to change the epidemiology of $\mathrm{PH}$ in this vulnerable population in Africa. Further studies including registries are urgently warranted, which should determine the incidence of HIV-induced PAH, and the relation between PAH and ART in HIV-infected individuals residing in Africa.

\section{Author affiliations}

${ }^{1}$ Department of Epidemiology and Public Health, Centre Pasteur of Cameroon, Yaoundé, Cameroon

${ }^{2}$ Bordeaux School of Public Health, University of Bordeaux, Bordeaux, France ${ }^{3}$ Faculty of Medicine and Biomedical Sciences, Department of Public Health, University of Yaoundé 1, Yaoundé, Cameroon

${ }^{4}$ Sickle Cell Disease Unit, Mother and Child Centre of the Chantal Biya Foundation, Yaoundé, Cameroon

${ }^{5}$ Faculty of Medicine and Biomedical Sciences, University of Yaoundé 1, Yaoundé, Cameroon

${ }^{6}$ Faculty of Medicine and Biomedical Sciences, School of Public Health, University of Queensland, Brisbane, Queensland, Australia

${ }^{7}$ Clinical Research Education, Networking and Consultancy (CRENC), Douala, Cameroon

${ }^{8}$ Infectious Diseases Unit, Yaoundé Central Hospital, Yaoundé, Cameroon ${ }^{9}$ Department of Medicine, Groote Schuur Hospital and University of Cape Town, Cape Town, South Africa

${ }^{10}$ Medical Diagnosis Center, Yaoundé, Cameroon

Contributors JJRB was involved in study conception and design. JJRB and LNA were involved in search strategy. JJRB and LNU were involved in study selection. JJRB and LNU were involved in data extraction. JJRB was involved in statistical analysis and drafting. JJRB and JRNN were involved in data interpretation. JJRB JRNN, LNA, LNU, SRNN, PSDS, JJNN and SK-S were involved in manuscript reviewing and revision. All authors approved the final version to publish.

Funding This research received no specific grant from any funding agency in the public, commercial or not-for-profit sectors.

Competing interests None declared.

Provenance and peer review Not commissioned; externally peer reviewed.

Data sharing statement No additional data are available.

Open Access This is an Open Access article distributed in accordance with the Creative Commons Attribution Non Commercial (CC BY-NC 4.0) license, which permits others to distribute, remix, adapt, build upon this work noncommercially, and license their derivative works on different terms, provided the original work is properly cited and the use is non-commercial. See: http:// creativecommons.org/licenses/by-nc/4.0/

\section{REFERENCES}

1. World Health Organization. HIV/AIDS: Fact sheet $\mathrm{N}^{\circ} 360$. Secondary HIV/AIDS: Fact sheet $N^{\circ} 3602015$ July 2015. http://www.who.int/ mediacentre/factsheets/fs360/en/

2. UNAIDS. 2014 Global Statistics. Secondary 2014 Global Statistics 2014. http://www.unaids.org/sites/default/files/media asset/ 20150714 FS MDG6 Report en.pdf

3. Simonneau G, Gatzoulis MA, Adatia I, et al. Updated clinical classification of pulmonary hypertension. J Am Coll Cardiol 2013;62 (Suppl 25):D34-41.

4. Hoeper MM, Bogaard HJ, Condliffe R, et al. Definitions and diagnosis of pulmonary hypertension. J Am Coll Cardiol 2013;62 (Suppl 25):D42-50.

5. Bigna JJ, Sime PS, Koulla-Shiro S. HIV related pulmonary arterial hypertension: epidemiology in Africa, physiopathology, and role of antiretroviral treatment. AIDS Res Ther 2015;12:36.
6. Correale M, Palmiotti GA, Lo Storto MM, et al. HIV-associated pulmonary arterial hypertension: from bedside to the future. Eur J Clin Invest 2015;45:515-28.

7. Crothers K, Huang L, Goulet JL, et al. HIV infection and risk for incident pulmonary diseases in the combination antiretroviral therapy era. Am J Respir Crit Care Med 2011;183:388-95.

8. Opravil M, Pechere M, Speich R, et al. HIV-associated primary pulmonary hypertension. A case control study. Swiss HIV Cohort Study. Am J Respir Crit Care Med 1997;155:990-5.

9. Moher D, Liberati A, Tetzlaff J, et al. Preferred reporting items for systematic reviews and meta-analyses: the PRISMA statement. Ann Intern Med 2009;151:264-9.

10. Viera AJ, Garrett JM. Understanding interobserver agreement: the kappa statistic. Fam Med 2005;37:360-3.

11. Hoy $\mathrm{D}$, Brooks $\mathrm{P}$, Woolf $\mathrm{A}$, et al. Assessing risk of bias in prevalence studies: modification of an existing tool and evidence of interrater agreement. J Clin Epidemiol 2012;65:934-9.

12. Vandenbroucke JP, von Elm E, Altman DG, et al, STROBE Initiative. Strengthening the Reporting of Observational Studies in Epidemiology (STROBE): explanation and elaboration. Int J Surg 2014;12:1500-24.

13. Higgins JP, Thompson SG, Deeks JJ, et al. Measuring inconsistency in meta-analyses. BMJ 2003;327:557-60.

14. Barendregt JJ, Doi SA, Lee YY, et al. Meta-analysis of prevalence. J Epidemiol Community Health 2013;67:974-8.

15. Cochran GW. The combination of estimates from different experiments. Biometrics 1954;10:101-29.

16. Higgins JP, Thompson SG. Quantifying heterogeneity in a meta-analysis. Stat Med 2002;21:1539-58.

17. Egger M, Davey Smith G, Schneider M, et al. Bias in meta-analysis detected by a simple, graphical test. BMJ 1997;315:629-34.

18. Ferrand RA, Desai SR, Hopkins C, et al. Chronic lung disease in adolescents with delayed diagnosis of vertically acquired HIV infection. Clin Infect Dis 2012;55:145-52.

19. Isiguzo GC, Okeahialam BN, Danbauchi SS, et al. Contributions of pulmonary hypertension to HIV-related cardiac dysfunction. Indian Heart J 2013;65:644-9.

20. Sliwa K, Carrington MJ, Becker A, et al. Contribution of the human immunodeficiency virus/acquired immunodeficiency syndrome epidemic to de novo presentations of heart disease in the Heart of Soweto Study cohort. Eur Heart J 2012;33:866-74.

21. Menanga AP, Ngomseu CK, Jingi AM, et al. Patterns of cardiovascular disease in a group of HIV-infected adults in Yaounde, Cameroon Cardiovasc Diagn Ther 2015;5:420-7.

22. Chillo $\mathrm{P}$, Bakari M, Lwakatare J. Echocardiographic diagnoses in HIV-infected patients presenting with cardiac symptoms at Muhimbili National Hospital in Dar es Salaam, Tanzania. Cardiovasc J Afr 2012;23:90-7

23. Quezada M, Martin-Carbonero L, Soriano V, et al. Prevalence and risk factors associated with pulmonary hypertension in HIV-infected patients on regular follow-up. AIDS 2012;26:1387-92.

24. Speich R, Jenni R, Opravil M, et al. Primary pulmonary hypertension in HIV infection. Chest 1991;100:1268-71.

25. Sitbon O, Lascoux-Combe C, Delfraissy JF, et al. Prevalence of HIV-related pulmonary arterial hypertension in the current antiretroviral therapy era. Am J Respir Crit Care Med 2008;177:108-13.

26. Kassaye SG, Katzenstein D. HIV/AIDS care and treatment in sub-Saharan Africa. AIDS Rev 2003;5:195-204.

27. Giuliano M, Vella S. Inequalities in health: access to treatment for HIV/AIDS. Ann Ist Super Sanita 2007;43:313-16.

28. Shors AR. The global epidemiology of HIV/AIDS. Dermatol Clin 2006:24:413-20.

29. Cleary S. Equity and efficiency in scaling up access to HIV-related interventions in resource-limited settings. Curr Opin HIV AIDS 2010:5:210-14.

30. World Health Organization. Guideline on when to start antiretroviral therapy and on pre-exposure prophylaxis for HIV. Secondary guideline on when to start antiretroviral therapy and on pre-exposure prophylaxis for HIV 2015. http://apps.who.int/iris/bitstream/10665/ 186275/1/9789241509565 eng.pdf

31. Jamieson D, Kellerman SE. The 909090 strategy to end the HIV Pandemic by 2030: can the supply chain handle it? J Int AIDS Soc 2016;19:20917.

32. Farber HW, Loscalzo J. Pulmonary arterial hypertension. N Engl J Med 2004;351:1655-65.

33. Torre D, Pugliese A. Impact of antiretroviral therapy among HIV-1-infected patients with pulmonary hypertension. Clin Infect Dis 2004;39:1549-50.

34. Thienemann F, Dzudie A, Mocumbi AO, et al. Rationale and design of the Pan African Pulmonary hypertension Cohort (PAPUCO) study: implementing a contemporary registry on pulmonary hypertension in Africa. BMJ Open 2014;4:e005950. 